\title{
Editorials
}

\section{Complete airway obstruction}

I $\mathrm{N}$ this issue of the Journal, McGuire and ElBeheiry report their experience with two patients who developed airway obstruction during attempted awake, endoscopic intubation; both were managed by surgical tracheostomy. ${ }^{1}$ This report raises issues regarding the management of the airway in spine-injured patients, or when unanticipated difficulties are encountered during airway intervention, as well as some related to decision-making during operating room crises.

The cases are eerily similar; both patients had an acute cervical spinal injury superimposed on chronic spinal disease. Both were sedated but arousable and breathing supplemental oxygen on transfer to the operating room although there is no mention as to whether it was continued. Both received iv sedation and glycopyrrolate. During flexible fibreoptic endoscopy, the first patient's airway became obstructed and desaturation occurred. Transtracheal oxygenation was established and the surgeon secured the airway with a tracheostomy. No attempt was made to insert a Combitube $^{\mathrm{TM}}$ or laryngeal mask airway, or to intubate the trachea, nor was retrograde intubation performed through the in situ transtracheal catheter. In the second patient, there was immediate deterioration in respiratory status following sedation and topicalization; the authors attempted flexible fibreoptic endoscopy but airway obstruction occurred. Following failed ventilation and failed direct laryngoscopy, the surgeon secured the airway with a tracheostomy. The authors conclude that complete upper airway obstruction developed in these patients because of a combination of heavy sedation and incomplete airway topicalization. I concur. Both patients benefitted from the immediate presence of a surgeon capable of rapidly performing a surgical tracheostomy. However, there are abundant reports which document that reliance on surgical tracheostomy to resolve anesthetic airway crises often results in a much more dismal outcome than noted in this report. ${ }^{2}$
Generally speaking, awake tracheal intubation is indicated when there is concern about the ability to ventilate the lungs after induction of anesthesia. It is also advised when intubation is anticipated to be difficult and airway protection with cricoid pressure is indicated, as protective forces cannot be sustained for long if difficulties are actually encountered. ${ }^{3}$ With respect to spine-injured patients, awake intubation is commonly performed to allow for assessment of neurological function after intubation and positioning, but before induction of general anesthesia. There is no evidence that awake intubation improves outcome in spine-injured patients but the practice continues to be favoured in many institutions, as it is in the authors'.

Awake intubation is facilitated by the administration of an antisialogogue; glycopyrrolate is favoured as it does not cross the blood brain barrier and thus does not cause sedation. A predictable and optimum drying effect is obtained with $i m$ administration $30-60 \mathrm{~min}$ before airway intervention. Supplemental oxygen administration (usually provided by nasal prongs) is recommended during awake intubation as it buffers the decrease in arterial oxygen saturation which occurs during airway intervention and which is worsened by the administration of sedatives. Sedation is best provided by the incremental titration of agents familiar to the attending physician to determined endpoints. Fentanyl and midazolam are commonly used and propofol is becoming more popular. Sedation causes a decreased airway calibre and airway obstruction; the most consistent site of obstruction is at the nasopharyngeal level where the soft palate becomes approximated to the posterior pharynx. ${ }^{4}$ In the event of deterioration in the airway with sedation, it may be necessary to reverse the effects of one or both of the agents rapidly and naloxone and flumazenil should be immediately available. Finally, the method of airway topicalization is usually operator-dependent; the operator should have a mechanism to determine that topicalization is adequate. 
In the setting of an unstable cervical spine, avoidance of secondary neurological injury resulting from airway interventions is a major concern. Untoward spinal movement may disrupt anatomic relationships, resulting in neural injury and neurologic deficit. All forms of airway interventions cause cervical spinal movement including chin lift, jaw thrust, placement of an oral or nasopharyngeal airway, mask ventilation and orotracheal intubation. ${ }^{5}$ There is no evidence that these movements cause new injury provided that interventions are executed with care. The head and spine movements which occur during different tracheal intubation techniques have been compared. Altering the choice of blade for the direct laryngoscope does not appear to affect movement. However, tracheal intubation using indirect techniques (Bullard laryngoscope and Augustine guide) cause less head and spinal extension than conventional laryngoscopes. Another indirect technique, using the lighted stylet, has been compared with that of the flexible endoscope for awake tracheal intubation in patients with cervical myelopathy or radiculopathy. ${ }^{6}$ There was no difference in the mean number of attempts required to achieve tracheal intubation although minor complications were more common after endoscope-facilitated intubation and the time to complete intubation was four times longer. Hung has also demonstrated the value of the lighted stylet as a rapid and efficient rescue technique for both recognized difficult intubations and unanticipated failed laryngoscopic intubation. ${ }^{7}$ It is tempting to infer from the above not only that indirect techniques which do not demand a direct glottic view will consistently produce less spinal movement than does the direct laryngoscope but will also function as efficient and effective salvage techniques in the event of unanticipated airway problems in spine-injured patients. Their use as first line techniques in this setting appears to be increasing, for good reason.

A number of innovative tools and strategies for difficult intubation have been advanced in recent years which address many of the factors which give rise to unanticipated difficulties during airway management. ${ }^{8}$ Interestingly, despite the apparent value of these techniques, anesthesia teachers seem reluctant to teach them and practitioners slow to adopt them. Koppel determined that instruction in difficult airway management is not offered by most US residency programs and that many airway management techniques are not taught at all.9 Similarly, Hung has determined that structured teaching of airway management does not occur in the majority of Canadian anesthesia teaching programs (Hung, personal communication). Rose reported that difficult laryngoscopy was most often managed with persistent attempts at direct laryngoscopy and that the use of alternative approaches to tracheal intubation was uncommon ( $1.9 \%$ of all tracheal intubations).${ }^{10}$ In these patients, there was a higher incidence of desaturation at induction, esophageal intubation, dental damage and unexpected ICU admissions. Alternatively, Hung noted that early conversion to an alternative technique (lighted stylet) in similar situations is typically rewarded with rapid tracheal intubation and that complications are rare. ${ }^{7}$ An early decision to employ an alternative technique in a difficult intubation due to poor direct laryngoscopic view may be associated with lower patient morbidity.

It is likely that over-reliance on airway techniques that are less than optimum for the task at hand is a riskenhancing behaviour which predisposes patients to morbidity and mortality, but such behaviour is common among anesthesiologists. Rosenblatt surveyed a random sample of the active membership of the American Society of Anesthesiologists. ${ }^{11}$ The survey presented difficult airway scenarios involving co-operative adult patients who required tracheal intubation and physicians were asked to identify their preferred management technique. Experienced practitioners tended to use higher risk induction techniques and were more likely to use the laryngeal mask airway in situations commonly agreed to be contraindicated. Again, the use of alternative devices including the Bullard scope, lighted stylet, and adjuncts was uncommon, occurring in $<5 \%$ of all scenarios. In the particular scenario of a patient with a history of previous difficult intubation, $60 \%$ of practitioners would induce general anesthesia and $59 \%$ would proceed with direct laryngoscopy. In about half of all instances, the following were not available for use in the institutions surveyed: lighted stylet, retrograde wire kit, Combitube, gum elastic bougie, Bullard laryngoscope. Seventeen percent of physicians surveyed did not even have access to the LMA. This despite its ease of use and affirmed efficacy as a salvage airway in the cannot intubate - cannot pentilate situation. ${ }^{12}$

Optimum management of unanticipated airway difficulties requires the ability to rapidly recognize, acknowledge, correctly analyze and respond to the problem with technical facility. Errant or delayed decision-making in this process may lead down an irremediable path to morbidity and mortality. Airway management can be considered to be "tightly coupled". What happens in one part (the airway) of the system directly affects other parts. Failed airway management may very quickly lead to catastrophic system failure (cardiorespiratory arrest). In order to cope with vast amounts of incoming information, anesthesi- 
ologists develop a large repertoire of minor response schema called into play by the occurrence of a specific event. The familiar event elicits a patterned response based on what has worked well in the past. Unfortunately, such patterned responses do not exist for rarer events such as a very difficult or impossible airway or failed ventilation. Pre-defined strategies for dealing with these predictable, albeit low frequency events become necessary.

A number of behaviour patterns frequently feature in investigations of accidents and are relevant to our understanding of airway mishaps. ${ }^{13}$ One is what is commonly referred to as "coning of attention". At a time when we most need to gather a wide range of information in order to make a good decision, we concentrate on one single source, choose the "first come, best preferred" solution and "solution fixation" develops. The second mechanism is "reversion under stress". Evidence shows that, under stress, recentlylearned behaviour patterns may be replaced by older, better-learned ones even if the older technique is not optimal for the task at hand. For example, in the event of an unanticipated difficult laryngoscopy with a perceived patient threat, recognition of the actual source of the problem (anterior larynx) may be missed; a well-learned intervention (direct laryngoscopy) may be persistently applied and re-applied (solution fixation), rather than intervening with a more optimum one (an indirect technique). Even as help arrives on scene, there may be a tendency to utilize sequentially the technique that has been from the outset, demonstrably inadequate. We've all been there.

Clearly, this report confirms that we must not only continue, but also enhance recent initiatives to evolve and practice organized approaches to airway problems, particularly those that were not anticipated. The teaching and application of newer techniques and technologies is integral to this process. Finally, we must acknowledge that patterns of behaviour, that are exceedingly common among ourselves, contribute to patient injury and we should work to remedy them.

\section{Obstruction complète des voies aériennes}

Dans le présent numéro du Journal, McGuire et ElBeheiry rendent compte de leur expérience auprès de deux patients qui ont développé une obstruction des voies aériennes pendant qu'on tentait de procéder à une intubation endoscopique vigile ; dans les deux cas, on a dû recourir à la trachéotomie. ${ }^{1} \mathrm{~L}$ 'article soulève des questions qui concernent soit l'intubation chez des sujets souffrant de lésion à la colonne vertébrale, soit l'intubation difficile imprévue ou encore des difficultés reliées à la prise de décision pendant des crises qui surviennent en salle d'opération.

Les cas sont étrangement similaires; les deux patients avaient une lésion cervicale aiguë de la colonne vertébrale surajoutée à une maladie vertébrale chronique. Ils ont tous les deux reçu une sédation, mais n'étaient qu'assoupis, et de l'oxygène supplémentaire lors de leur transfert vers la salle d'opération. On ne sait si cela s'est poursuivi. On leur a administré de la sédation $i v$ et du glycopyrrolate. Pendant la fibroscopie, il y a eu une obstruction des voies aériennes suivie d'une désaturation chez le premier patient. On a établi l'oxygénation transtrachéale et le chirurgien a maintenu la perméabilité des voies respiratoires par une trachéotomie. On n'a pas tenté d'insérer un Combitube ${ }^{\mathrm{TM}}$ ou un masque laryngé, ni de procéder à une intubation endotrachéale ou à une intubation rétrograde au moyen du cathéter transtrachéal in situ. Chez le second patient, il y a eu une détérioration immédiate de la respiration après la sédation et la pulvérisation d'anesthésique ; la tentative des auteurs de procéder à une fibroscopie s'est soldée par l'obstruction des voies respiratoires. À la suite de cet échec de la ventilation et de la laryngoscopie directe, le chirurgien a préservé les voies respiratoires par une trachéotomie. Les auteurs concluent que l'obstruction complète des voies respiratoires supérieures est survenue chez ces patients à cause de la combinaison d'une sédation lourde et d'une pulvérisation incomplète des voies respiratoires. Je suis d'accord. Les deux patients ont bénéficié de la présence, sur place, d'un chirurgien capable de procéder rapidement à une trachéotomie. Cependant, d'abondants articles qui font état de ce recours à la trachéotomie pour supprimer les crises respiratoires lors de l'anesthésie montrent que les résultats sont souvent beaucoup moins heureux que ceux qui sont rapportés ici. ${ }^{2}$

Généralement, l'intubation endotrachéale vigile est recommandée lorsqu'on appréhende des difficultés de ventilation pulmonaire après l'induction de l'anesthésie. C'est à conseiller également quand on craint une intubation difficile et que la protection des voies respiratoires nécessite une pression cricoïde, puisque cette pression protectrice ne peut être maintenue longtemps. ${ }^{3}$ En ce qui concerne les patients atteints de lésion vertébrale, l'intubation vigile est couramment réalisée pour permettre l'évaluation de la fonction neurologique qui suit l'intubation et le positionnement, 
mais qui précède l'induction de l'anesthésie générale. Il n'existe aucune preuve que l'intubation vigile améliore l'évolution des patients atteints de lésion vertébrale, mais cette pratique est toujours favorisée dans maintes institutions, dont celle des auteurs.

L'intubation vigile est facilitée par l'administration d'un antisialogogue ; on utilise surtout le glycopyrrolate vu qu'il ne traverse pas la barrière hémato-encéphalique et, par conséquent, ne cause pas de sédation. Un effet maximal prévisible a été obtenu avec l'administration im 30-60 min avant l'intubation. L'addition d'oxygène supplémentaire (habituellement par voie nasale) est recommandée pendant l'intubation vigile, car elle atténue la baisse de saturation artérielle en oxygène qui survient pendant l'intubation et qui est aggravée par l'administration de sédatifs. La sédation est mieux assurée par le titrage incrémentiel d'agents familiers au médecin traitant pour des cibles déterminées. Le fentanyl et le midazolam sont ordinairement utilisés, mais le propofol est de plus en plus connu. La sédation diminue le calibre des voies aériennes et cause de l'obstruction ; c'est le nasopharynx qui constitue le plus souvent le site de l'obstruction, à l'endroit où le palais mou se rapproche de la paroi postérieure du pharynx. ${ }^{4}$ Si une détérioration des voies aériennes survient lors de la sédation, il peut être nécessaire de renverser rapidement les effets de l'un des agents, ou des deux, et le naloxone et le flumazénil devraient être immédiatement disponibles. Enfin, ajoutons que la pulvérisation des voies aériennes dépend habituellement de l'opérateur; ce dernier devrait pouvoir déterminer l'efficacité de la pulvérisation.

Dans le contexte d'une colonne cervicale instable, nous désirons surtout éviter une lésion neurologique secondaire qui pourrait résulter de l'intubation. Des mauvais mouvements peuvent rompre les relations anatomiques de la colonne, causant ainsi une lésion neurale et un déficit neurologique. Toutes les formes d'interventions sur les voies aériennes entraînent des mouvements de la colonne y compris la traction sur le menton et la subluxation de la mâchoire, le placement d'un tube oro- ou nasopharyngien, la ventilation par masque ou l'intubation avec un tube orotrachéal. ${ }^{5}$ Rien ne prouve que ces mouvements causent de nouvelles lésions pourvu que les interventions soient exécutées avec soin. Les mouvements de la tête et de la colonne qui surviennent pendant l'utilisation de différentes techniques d'intubation trachéales ont été comparés. Choisir une autre lame pour le laryngoscope direct n'a pas, semble-t-il, d'effet sur le mouvement. Cependant, l'intubation endotrachéale selon des techniques indirectes (le laryngoscope Bullard et le guide Augustine) provoque moins d'extension de la tête et de la colonne que l'emploi des laryngoscopes traditionnels. Une autre technique indirecte, avec le stylet lumineux, a été comparée à l'emploi de l'endoscope flexible pour l'intubation vigile chez des patients atteints de myélopathie cervicale ou de radiculopathie. ${ }^{6}$ Il n'y avait pas de différence du nombre moyen d'essais nécessaires à l'intubation endotrachéale quoique des complications mineures ont été plus fréquentes après l'intubation facilitée par l'endoscope et que cette démarche a demandé quatre fois plus de temps. Hung a aussi démontré la valeur du stylet lumineux en tant que technique de secours rapide et efficace dans les cas d'intubations difficiles reconnues et d'échec inattendu de l'intubation laryngoscopique. ${ }^{7}$ On est porté à conclure de tout cela que non seulement les techniques indirectes qui ne demandent pas de visualiser directement la glotte vont, de façon évidente, produire moins de mouvement de la colonne que le laryngoscope direct, mais qu'elles vont aussi se présenter comme des techniques de secours efficaces dans les cas de problèmes inattendus d'intubation chez des patients atteints de lésions de la colonne. Leur utilisation comme techniques de première ligne dans cette situation semble s'accroître avec raison.

Nombre de stratégies et d'outils innovateurs ont été mis au point au cours des dernières années pour palier les difficultés d'intubation. Ces innovations tiennent compte de nombreux facteurs qui donnent lieu à des difficultés non anticipées pendant l'intubation. ${ }^{8}$ Il est intéressant de noter que, malgré la valeur apparente de ces techniques, les professeurs d'anesthésie semblent réticents à les enseigner et les praticiens, lents à les adopter. Koppel a établi que l'enseignement de la gestion des difficultés d'intubation n'est pas offert dans la plupart des programmes de résidence américains et que beaucoup de techniques d'intubation ne sont pas du tout enseignées. ${ }^{9}$ De même, Hung a découvert que l'enseignement structuré de l'intubation ne se retrouve pas dans la majorité des programmes d'anesthésie canadiens (Hung, communication personnelle). Rose a souligné que devant une intubation difficile, on a le plus souvent recours à des essais persistants au moyen de la laryngoscopie directe et que l'emploi de méthodes de rechange est rare (1,9\% de toutes les intubations). ${ }^{10}$ Chez les patients ainsi traités, il y a une plus grande incidence de désaturation à l'induction, d'intubation oesophagienne, de dommages dentaires et d'admissions imprévues à l'USI. Par ailleurs, Hung a noté que le recours hâtif à une technique de remplacement (stylet lumineux) dans des circonstances similaires est normalement récompensé par une intubation endotrachéale rapide dont les complications sont rares. ${ }^{7} \mathrm{La}$ 
décision précoce d'employer une autre technique quand l'intubation est difficile à cause d'une vision pauvre par laryngoscopie directe peut réduire la morbidité chez les patients.

Il semble que trop se fier à des techniques qui ne sont pas optimales pour la tâche à faire soit un comportement risqué qui prédispose les patients à la morbidité et à la mortalité, pourtant cette attitude est fréquente parmi les aneshésiologistes. Rosenblatt a enquêté auprès d'un échantillon aléatoire de membres actifs de l'American Society of Anesthesiologists. ${ }^{11}$ L'enquête a présenté différents scénarios d'intubation difficile où le patient est un adulte coopératif qui a besoin d'intubation endotrachéale et où le médecin doit identifier sa technique préférée. Les médecins expérimentés avaient tendance à utiliser des techniques d'induction à plus grand risque et étaient plus susceptibles d'employer le masque laryngé dans des situations où on s'accorde généralement à le déconseiller. De plus, l'usage de dispositifs de rechange incluant le laryngoscope Bullard, le stylet lumineux et des accessoires, n'était pas répandu, apparaissant dans $<5 \%$ de tous les scénarios. Dans le cas particulier d'un patient chez qui l'intubation a déjà été difficile, $60 \%$ des médecins induisaient l'anesthésie générale et $59 \%$ utilisaient la laryngoscopie directe. Dans la moitié des cas environ, les appareils suivants n'étaient pas disponibles dans les institutions sous enquête : le stylet lumineux, la trousse pour intubation rétrograde, le Combitube $^{\text {rm }}$, la bougie en caoutchouc, le laryngoscope Bullard. Dix-sept pour cent des médecins de l'enquête n'avaient même pas accès au ML. Et cela en dépit de son usage facile et de son efficacité reconnue comme mesure d'intubation de secours dans les situations où on ne peut ni intuber ni ventiler. ${ }^{12}$

La gestion optimale des difficultés d'intubation imprévues exige la compétence à reconnaître rapidement la situation, à l'analyser correctement et à y faire face avec une facilité d'exécution technique. Errer ou retarder la prise de décision dans les circonstances peut conduire irrémédiablement vers la morbidité et la mortalité. La prise en charge des voies aériennes peut être considérée comme un "couplage serré». Ce qui survient dans une partie (les voies aériennes) du système affecte directement les autres parties. Une défaillance dans le traitement des voies aériennes peut conduire très rapidement à une défaillance catastrophique du système (arrêt cardiorespiratoire). Dans le but de mieux absorber la somme d'informations nouvelles, les anesthésiologistes élaborent un grand répertoire de petits schémas de réponses appelées à jouer leur rôle selon l'occurrence d'un événement spécifique. Un événement familier déclenche un modèle de réponse basé sur ce qui a bien fonctionné dans le passé. Malheureusement, ces modèles de réponses n'existent pas pour des incidents rares comme une intubation très difficile ou impossible ou une défaillance de la ventilation. Des stratégies prédéfinies permettant de faire face à ces incidents prévisibles, quoique peu fréquents, sont devenues nécessaires.

Certaines attitudes sont fréquemment mises en relief lors d'investigations sur des accidents et sont pertinentes pour notre compréhension des problèmes d'intubation. ${ }^{13}$ L'une d'elle est couramment appelée la «limitation de l'attention». Au moment où nous avons le plus besoin de rassembler beaucoup d'informations afin de prendre une bonne décision, nous nous concentrons sur une seule source, choisissons la solution "préférée, première arrivée» et se développe alors la «fixation sur la solution». Le second mécanisme est la «régression sous la pression». La preuve est faite que, sous tension, les compétences récemment acquises peuvent être remplacées par des techniques plus anciennes, mieux apprises, même si elles ne sont pas optimales pour la tâche présente à accomplir. Par exemple, dans le cas d'une laryngoscopie difficile imprévue où le patient est menacé, on peut manquer de reconnaître la véritable source du problème (la paroi antérieure du larynx); une intervention bien apprise (la laryngoscopie directe) peut être appliquée à répétition (fixation sur la solution), plutôt qu'une intervention plus optimale (une technique indirecte). Même quand une aide entre en scène, on peut avoir tendance à utiliser les séquences de la technique qui était, depuis le début, manifestement inappropriée. Nous sommes tous passés par là.

A l'évidence, cet article confirme que nous devons non seulement continuer, mais aussi stimuler les initiatives récentes pour faire évoluer et pratiquer des méthodes organisées de résolution des problèmes d'intubation, surtout ceux qui sont imprévus. L'enseignement et l'application de nouvelles techniques et technologies font partie intégrante de cette pratique. Finalement, nous devons reconnaître que par des modèles de comportements qui sont excessivement fréquents parmi nous, nous faisons partie du problème du patient et que nous devons travailler à corriger la situation.

\section{References}

1 McGuire G, El-Beheiry $H$. Complete upper airway obstruction during awake fibreoptic intubation in patients with unstable cervical spine fractures. Can J Anaesth 1999; 46: 176-8.

2 Jones $D H$, Cohle $S D$. Unanticipated difficult airway secondary to lingual tonsillar hyperplasia. Anesth Analg 1993; 77: 1285-8. 
3 Meek T, Vincent A, Duggan JE. Cricoid pressure: can protective force be sustained? $\mathrm{Br}$ J Anaesth 1998; 80: 672-4.

4 Boidin, MP. Airway patency in the unconscious patient. Br J Anaesth 1985; 57: 306-10.

5 Hastings RH. Airway management of patients with cervical spine injury. Prob Anesth 1997; 9: 25-37.

6 Saba $A K$, Higgins $M$, Walker $G$, Badr A, Berman $L$. Comparison of awake endotracheal intubation in patients with cervical spine disease: the lighted intubating stylet versus the fiberoptic bronchoscope. Anesth Analg 1998; 87: 477-9.

7 Hung OR, Pytka S, Morris I, Murpby M, Stewart RD. Lightwand intubation: II. Clinical trial of a new lightwand for tracheal intubation in patients with difficult airways. Can J Anaesth 1995; 42: 826-30.

8 Crosby ET, Cooper RM, Douglas MJ, et al. The unanticipated difficult intubation with recommendations for management. Can J Anaesth 1998; 45: 757-76.

9 Koppel $J N$, Reed AP. Formal instruction in difficult airway management. A survey of anesthesiology residency programs. Anesthesiology 1995; 83: 1343-6.

10 Rose $D K$, Coben $M M$. The airway: problems and predictions in 18,500 patients. Can J Anaesth 1994; 41 : 372-83.

11 Rosenblatt WH, Wagner PJ, Ovassapian A, Kain ZN. Practice patterns in managing the difficult airway by anaesthesiologists in the United States. Anesth Analg 1998; 87: 153-7.

12 Parmet JL, Colonna-Romano P, Horrow JC, Miller F, Gonzales $J$, Rosenberg $H$. The laryngeal mask airway reliably provides rescue ventilation in cases of unanticipated difficult tracheal intubation along with difficult mask ventilation. Anesth Analg 1998; 87: 661-5.

13 Allnutt MF. Human factors in accidents. $\mathrm{Br} \mathrm{J}$ Anaesth 1987; 59: 856-64. 